\title{
Giant Fine Structure Splitting of the Bright Exciton in a Bulk $\mathrm{MAPbBr}_{3}$ Single Crystal
}

\author{
Michał Baranowski, ${ }^{\dagger \dagger}$ Krzysztof Galkowski, ${ }^{\dagger} \uparrow$ Alessandro Surrente, ${ }^{\dagger}$ Joanna \\ Urban, ${ }^{\dagger}$ Łukasz Kłopotowski, ${ }^{\S}$ Sebastian. Maćkowski, $₫$ Duncan Kennedy \\ Maude, ${ }^{\dagger}$ Rim Ben Aich," Kais Boujdaria," Maria Chamarro, ${ }^{\perp}$ Christophe \\ Testelin, ${ }^{\perp}$ Pabitra Nayak," Markus Dollmann,"\# Henry James Snaith," Robin \\ Nicholas, ${ }^{*}, \#$ and Paulina Plochocka*,t,ł \\ $\dagger$ Laboratoire National des Champs Magnétiques Intenses, UPR 3228, \\ CNRS-UGA-UPS-INSA, Grenoble and Toulouse, France \\ $\ddagger$ Department of Experimental Physics, Faculty of Fundamental Problems of Technology, \\ Wroclaw University of Science and Technology, Wroclaw, Poland \\ IInstitute of Physics, Faculty of Physics, Astronomy and Informatics, Nicolaus Copernicus \\ University, 5th Grudziadzka St., 87-100 Torun, Poland \\ §Institute of Physics, Polish Academy of Sciences, al. Lotnikow 32/46, 02-668 Warsaw, \\ Poland \\ ||Laboratoire de Physique des Matériaux : Structure et Propriétés, Faculté des Sciences de \\ Bizerte, Université de Carthage, 7021 Zarzouna, Bizerte, Tunisia \\ $\perp$ Sorbonne Université, CNRS-UMR 7588, Institut des NanoSciences de Paris, INSP, 4 \\ place Jussieu, F-75005, Paris, France \\ \# University of Oxford, Clarendon Laboratory, Parks Road, Oxford, OX1 3PU, United \\ Kingdom \\ E-mail: robin.nicholas@physics.ox.ac.uk; paulina.plochocka@lncmi.cnrs.fr \\ Phone: 01865 (2)72250; +330562172862
}

\section{Abstract}

Exciton fine structure splitting in semiconductors reflects the underlying symmetry of the crystal and quantum confinement. Since the latter factor strongly enhances the exchange interaction, most work has focused on nanostructures. Here, we report on the first observation of the bright exciton fine structure splitting in a bulk semiconductor crystal, where the impact of quantum confinement can be specifically excluded, giving access to the intrinsic properties of the material. Detailed investigation of the exciton photoluminescence and reflection spectra of a bulk methylammonium lead tribromide single crystal reveals a zero magnetic field split- ting as large as $\sim 200 \mu \mathrm{eV}$. This result provides an important starting point for the discussion of the origin of the large bright exciton fine structure observed in perovskite nanocrystals.

KEYWORDS:Exciton, Fine Structure Splitting, Perovskite, Bulk, Photoluminescence, Reflectance

In an ideally pure semiconductor, the lowest energy electronic excitation is a bound electronhole pair (exciton). The exchange interaction between electron and the hole spins lifts the degeneracy between dark singlet and bright multiplet excitonic states producing a fine structure. The physics of the fine structure splitting (FSS) has been the subject of intense in- 
vestigation, 113 since it can be used for quantum logic, $\frac{14}{14}$ and wave function control. $\frac{15}{15}$ Moreover, the ability to control the FSS is essential to fabricate the efficient entangled photon sources ${ }^{16}$ required for quantum teleportation, ${ }^{21}$ quantum communication, 22 and quantum cryptography. 23

The pattern of excitonic states produced by the exchange interaction strongly depends on the symmetry of the system. In structures with sufficiently low symmetry, the degeneracy of the bright states should be completely lifted. However, in general, it is expected that the bright exciton FSS is much smaller than the brightdark state splitting, $\underline{6}$ which is typically a few tens of $\mu \mathrm{eV}$ in common inorganic semiconductors. ${ }^{24}$ Since the symmetry breaking is generally also small (or even absent), the bright exciton FSS has never been observed in any bulk semiconductor. In contrast, the bright exciton FSS is commonly observed in semiconductor nanostructures ${ }^{1 / 8}$ where quantum confinement greatly enhances the exchange interaction and the symmetry is strongly broken by the anisotropic quantum confinement.

Recently, different theoretical models predict that the low symmetry of lead-halide perovskites in the orthorombic phase should result in a significant bright exciton fine structure splitting. While there is no consensus about the origin of this phenomenon, which can result from the Rashba effect $25 \sqrt{27}$ or interplay of exchange interaction and crystal field, $\stackrel{28}{31}$ all of those predictions make lead halide perovskites a promising starting point to search for the elusive bright exciton FSS in bulk semiconductors.

Here, we report that indeed the bright exciton FSS can be observed in a high quality bulk $\mathrm{MAPbBr}_{3}$ single crystal in which all the extrinsic sources of symmetry breaking and quantum confinement enhancement can be specifically excluded. Therefore our work constitutes an important base for further discussions of the intrinsic origin of exciton FSS in perovskite crystals. By means of detailed magneto-optical spectroscopy, we reveal the FSS of the exciton 1s transition with a splitting as large as $200 \mu \mathrm{eV}$. We show that the observed value can be reasonably explained by the recent model proposed in refs. $\frac{29130}{2}$ with parameters extracted from our experimental studies.

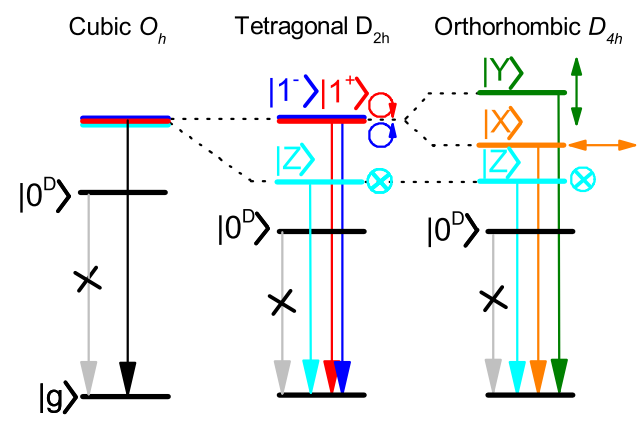

Figure 1: Schematic showing the exciton fine structure for the different crystal structures of perovskite and expected circular or linear polarization of the optical transitions. The presented scheme takes into account the exciton symmetry in the cubic $\left(O_{h}\right)$, tetragonal $\left(D_{2 h}\right)$ and orthorombic $\left(D_{4 h}\right)$ cases, the crystal field and the exchange interaction. ${ }^{28 \mid 29}$ Including Rashba splittings could lead to further symmetry reduction and to the bright states being lower in energy than their dark counterpart. 25

In lead-halide perovskites, the conduction and valence bands are built mainly from cationic $\mathrm{Pb}$ orbitals. Crucially, the valence band is built from s-like orbitals, while the conduction band is built from p-like orbitals. $32+35$ Strong spinorbit coupling in the conduction band ${ }^{[32}$ splits the electron states with total angular momentum $J=3 / 2$ (upper band) and $J=1 / 2$ (lower band). Therefore, the band-edge exciton can be in one of four degenerate states: $\left|0_{D}\right\rangle$ with zero total angular momentum, $J^{e x c}=0$, and three states $|Z\rangle,| \pm 1\rangle$ with $J^{e x c}=1$ and $z$ components of angular momentum $J_{z}^{e x c}=0$ or $J_{z}^{e x c}= \pm 1$ respectively.

In a cubic crystal, the exchange interaction splits the dark, $J^{e x c}=0$, exciton state from the triply degenerate bright, $J^{e x c}=1$, states. Depending on temperature, the lead-halide perovskite crystal can be in a cubic, or tetragonal or orthorhombic phase. ${ }^{36 / 37}$ In the latter two phases, crystal field together with exchange interaction lifts the degeneracy of bright states. 28|29|38 40 In the tetragonal phase, the bright exciton levels split into two degenerate $| \pm 1\rangle$ states, which couple to circularly (elec- 

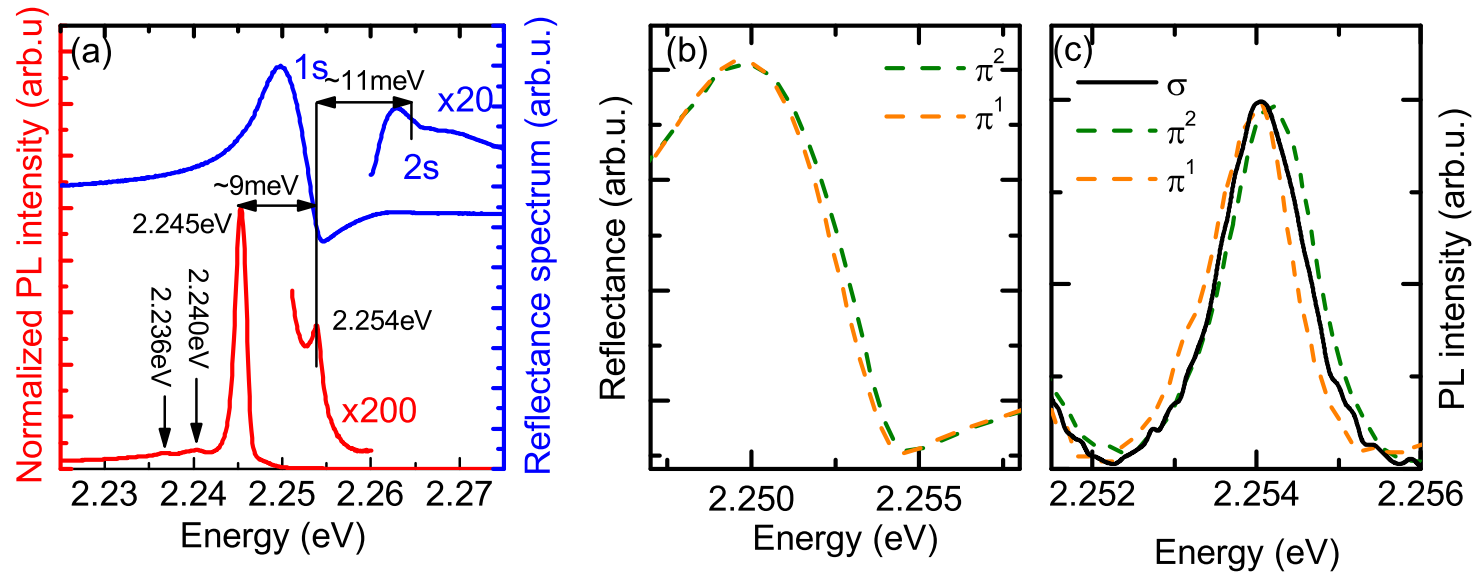

Figure 2: PL (red line) and reflectance (blue line) spectra measured at $T=1.5 \mathrm{~K}$. The 1 s excitonic transition is observed in PL and both the 1 s and 2 s states can be identified in reflectance. The strong PL emission $9 \mathrm{meV}$ below the 1s transition (with no corresponding feature in reflectance) is assigned to bound exciton emission. (b) Reflectance spectrum measured in a linear polarization basis for two orthogonal polarizations. (c) Two linear orthogonally polarized components of PL spectra (dashed lines) together with PL spectra measured in circular polarization basis. For clarity the PL background has been subtracted.

tric vector in $x y$ plane) polarized light and a $|Z\rangle$ state, which couples to linearly (electric vector along $z$ direction) polarized light. The further symmetry reduction in the orthorhombic (low temperature) phase lifts the degeneracy of the $| \pm 1\rangle$ states resulting in symmetric and antisymmetric states $|X\rangle=\left(\left|1^{+}\right\rangle+\left|1^{-}\right\rangle\right) / \sqrt{2}$, $|Y\rangle=\left(\left|1^{+}\right\rangle-\left|1^{-}\right\rangle\right) / \sqrt{2}$, which couple to the two other linearly orthogonal polarizations of light, respectively. The expected fine structures of excitons in different crystallographic phases are summarized in Figure 1 .

Very recently, bright exciton FSS (in the range of few hundred $\mu \mathrm{eV}$ up to $1-2 \mathrm{meV}$ ) has been reported for lead halide perovskite nanocrystals. $\frac{25|26| 28|29| 40}{44}$ However it is not clear if the observed splitting is related only to the crystal field and exchange interaction $28|29| 40 \mid 41]$ or if it is enhanced or driven by the Rashba effect. ${ }^{25126}$ Moreover, in nanocrystals one has to worry about possible contributions of anisotropic quantum confinement to the FSS $11 / 29 \sqrt[40 \mid 45]{47}$ or a surface enhancement of the Rashba effect. $\stackrel{489}{ }$ The significant variation in the size of bright exciton FSS observed in the nanocrystals 25141 indicates that the fine structure splittings in perovskites can be strongly affected by extrinsic contributions. This hinders the determination of the intrinsic properties of perovskite crystals, which are required to provide a starting point for the discussion of the origin of the large bright exciton fine structure splitting. Moreover, the random orientation of the nanocrystals, together with the presence of high temperature phases even at cryogenic temperatures, ${ }^{[50}$ further complicates the interpretation of the exciton FSS.

The few $\mathrm{mm}$ by few $\mathrm{mm}$ single $\mathrm{MAPbBr}_{3}$ crystal were prepared as reported for $\mathrm{MAPbBr}_{3}$ in ref.51 Briefly, MABr (from Dyesol) and $\mathrm{PbBr}_{2}$ (Sigma-Aldrich) salts were added to dimethyl formamide (DMF) to prepare a $1 \mathrm{M}$ solution. Formic acid $(\sim 40 \mu \mathrm{l} / \mathrm{ml})$ was added to the above solution followed by a filtration with a 0.45 micron filter. The solution incubated at $55^{\circ} \mathrm{C}$ produced multiple seed crystals of $(\sim 500 \mu \mathrm{m})$. The seed crystals (which are single crystalline as we have used them for XRD characterization $^{51}$ ) were collected and used as seed for the growth of $\mathrm{mm}$ size crystals where the DMF solution has less formic acid. Each seed produced only one large crystal and the number of large crystals were controlled by the number of seeds added. Namely the filtered solution was poured into a glass vial and a cleaned glass slide was placed at the bottom 
of the vial. A few small seeds $(\sim 500 \mu \mathrm{m})$ of $\mathrm{MAPbBr}_{3}$ crystal were carefully placed on the glass slide. The glass vial was then incubated at $\sim^{\circ} 55 \mathrm{C}$ to produce the large $\mathrm{MAPbBr}_{3}$ crystals of size $2 \times 2 \times 1 \mathrm{~mm}^{3}$. The crystals were collected when the solution is still hot $\left(\sim 55^{\circ} \mathrm{C}\right)$ and preserved in chlorobenzene. Before the measurements, the crystals were blow dried with a $\mathrm{N}_{2}$ gun. The measurements were performed on the largest facet of the crystal.

The magnetic field measurements were performed in a cryofree split coil (up to $7 \mathrm{~T}$ ) equipped with a variable temperature insert $(1.5-300 \mathrm{~K})$. The optical access to the sample was through quartz B optical windows. The white light used for the reflection studies was provided by a tungsten lamp. For the PL measurements the sample was excited using a $405 \mathrm{~nm}$ continuous wave (CW) laser with a power of $100 \mu \mathrm{W}$. The PL and reflection measurement were performed in the Faraday geometry where the excitation beam or white light was directed onto the sample through a nonpolarizing beam splitter and focused on the sample by a $20 \mathrm{~cm}$ focal length lens. The signal was collected by the same lens and dispersed by a $0.5 \mathrm{~m}$ long spectrometer with a 1800 groove/mm grating. The dispersed light was detected using a nitrogen cooled CCD camera. The polarization of the signal was analyzed using a broad band half wave or quarter wave plate and linear polarizer.

All our measurements have been performed in the low temperature orthorhombic phase(which was confirmed by temperature dependence of $\mathrm{PL}$ presented in SI). The macroPL and reflectance spectrum of the $\mathrm{MAPbBr}_{3}$ crystal taken at $T=1.5 \mathrm{~K}$ are presented in Figure 2(a). The PL spectrum (red curve) is dominated by a sharp feature at $2.245 \mathrm{eV}$ with a $1.5 \mathrm{meV}$ full width at half maximum (FWHM). In reflectance, in addition to the strong dispersive resonance observed for the 1 s free exciton transition, a much weaker feature corresponding to the $2 \mathrm{~s}$ free exciton state is observed on the high energy side (see expanded $\times 20$ curve). For the dispersive reflectance line shape the resonance position, indicated by the vertical black dash/dotted lines, corresponds to the maximum slope.

The $1 \mathrm{~s}$ and $2 \mathrm{~s}$ states are separated by $\simeq$ $11 \mathrm{meV}$, consistent with an exciton binding energy $R_{y}^{*} \simeq 14.5 \mathrm{meV}$ (assuming a hydrogenic model). This value of exciton binding energy is in good agreement with those reported previously. ${ }^{[52}$ The dominant PL peak is red-shifted by $\simeq 9 \mathrm{meV}$ with respect to the resonance observed in the reflectance spectrum. Furthermore, on the high energy side of the PL spectrum there is a relatively weak peak (see expanded $\times 200$ curve) at the same energy as the 1s excitonic transition observed in reflectance. Therefore, we attribute the strong PL emission to bound states $^{37}$ (see also extended dissuasion in SI), and the small PL feature on the high energy side to the 1 s free exciton emission.

Moreover two weak low energy peaks separated by about $5 \mathrm{meV}$ and $9 \mathrm{meV}$ from the strongest PL peak can be observed. Such peaks have already been observed in $\mathrm{CsPbBr}_{3}$ perovskite nanocrystals and attributed to TO phonon replicas. ${ }^{[2]}$ Interestingly the separation between the first phonon replica and the 1s excitonic peak is $14 \mathrm{meV}$ which is also very close to the effective energy of the LO phonon reported for $\mathrm{MAPbBr}_{3}$ crystals. $\stackrel{53}{ }$ Therefore the lowest energy bands can be attributed to phonon replicas of the bound and free exciton transitions.

Reflectance and PL spectra measured in a linear polarization basis are shown in Figure $2(b, c)$. The energy shift between the orthogonal linear polarizations $\pi^{1}$ and $\pi^{2}$, clearly visible in both PL and reflectance, is the smoking gun signature of the bright exciton FSS in a bulk crystal.

Before discussing in detail the zero magnetic field FSS, we present magneto-optical data taken in the Faraday configuration, which unambiguously demonstrates the free excitonic nature of the high energy PL feature. Figure3(a) shows reflectance spectra measured for the two opposite circular polarizations $\sigma^{1}$ and $\sigma^{2}$ at 0 and $7 \mathrm{~T}$ magnetic field. The expected shift of the $\sigma^{1}$ and $\sigma^{2}$ components in magnetic field is clearly seen. To determine precisely the splitting of the $\sigma^{1}$ and $\sigma^{2}$ transition as a function of magnetic field we divide them by the zero field spectrum (see Figure $3(\mathrm{~b})$ ). The ra- 

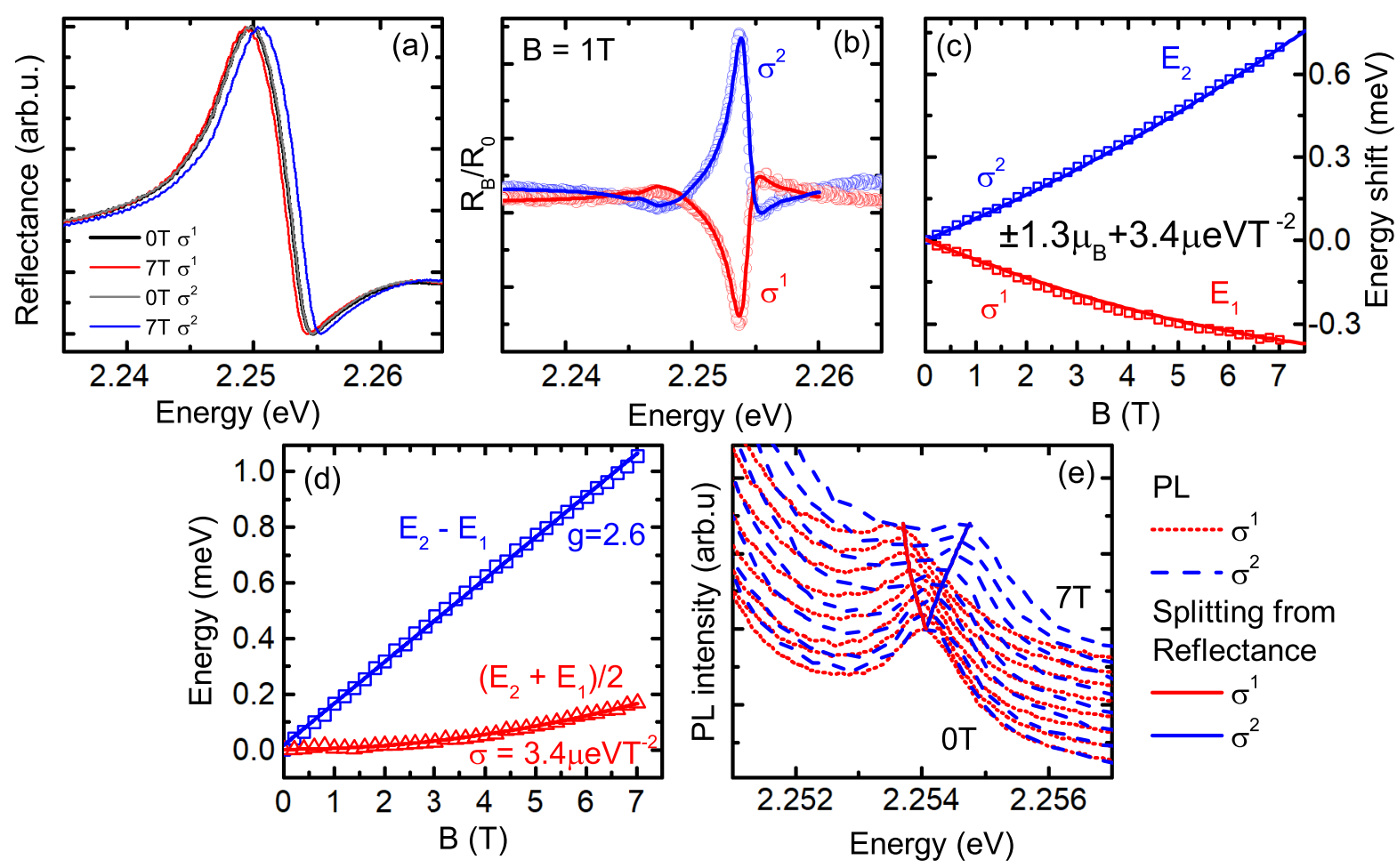

$P L$

.......... $\sigma^{1}$

$--\sigma^{2}$

Splitting from

Reflectance

$-\sigma^{1}$

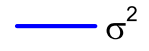

Figure 3: (a) $\sigma^{1}$ and $\sigma^{2}$ components of the reflectance spectrum measured at zero (black and gray curve) and $7 \mathrm{~T}$ (red and blue). (b) Reflectance spectrum at $1 \mathrm{~T}$ divided by the zero field spectra (open points) together with a fit using equation (1). (c) Shift of the $\sigma^{1}$ and $\sigma^{2}$ components as a function of magnetic field. (d) Determined Zeeman splitting (blue squares) and diamagnetic shift (red triangles) together with linear and quadratic fit, respectively. (e) Comparison of the reflectance shift with the PL spectra feature assigned to free exciton emission.

tioed spectra have a characteristic sharp feature with an amplitude and width which increase with the size of the energy shift. As the shape of the reflectance spectra are almost unchanged in magnetic field, the shift can be measured by fitting to the ratioed spectra by varying the shift between the spectra in

$$
\frac{R_{B}(E)}{R_{0}(E)} \propto \frac{R_{0}(E+\Delta E)}{R_{0}(E)},
$$

where $R_{B}(E)$ and $R_{0}(E)$ are reflectance spectra in magnetic field and at zero field and $\Delta E$ is the shift of the reflectance resonance in magnetic field. More details about fitting procedure can be found in the supplementary information. The fits, shown as solid lines Figure 3(b), are in excellent agreement with the experimental data. This method allows us to precisely determine the shift of the exciton transition even at very low magnetic field and avoids having to model the reflection line shape.

The energy shift of the $\sigma^{1}$ and $\sigma^{2}$ transitions and the resulting Zeeman splitting and diamagnetic shift are summarized in Figure $3(\mathrm{c}, \mathrm{d})$. Fitting the splitting of the $\sigma^{1}$ and $\sigma^{2}$ features with $E_{2}-E_{1}=g \mu_{B} B$ (see Figure $3(\mathrm{~d}))$ gives $g=2.6 \pm 0.1$, in agreement with the previously reported value. ${ }^{38}$ The diamagnetic shift is extracted by fitting the average behavior in field $\left(\sigma B^{2}=\left(E_{2}+E_{1}\right) / 2\right)$, giving $\sigma=(3.4 \pm 0.1) \mu \mathrm{eVT}^{-2}$ with a resulting exciton Bohr radius of $\simeq 3.8 \mathrm{~nm}$ and effective mass of $\mu=0.185 m_{0}$. Finally, we compare the behavior of the exciton transitions in magnetoreflectance with the behavior of the corresponding 1s features in PL (broken lines Figure 3(e))). The 1s PL feature shifts in exactly the same way as in the reflectance data, plotted in solid lines.

Two in-plane bright excitons should be linearly polarized and energetically separated (see also discussion in supplementary information). 


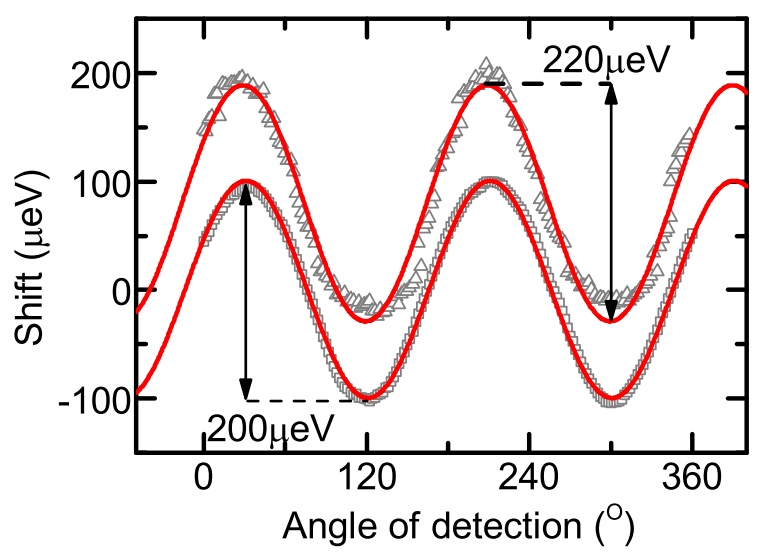

Figure 4: (a) Shift of the reflectance resonance (open squares) and PL peak (open triangles) as a function of detection angle of linear polarization. For clarity, the PL data is shifted vertically by $100 \mu \mathrm{eV}$. The red lines are fits using a $\sin ^{2} \theta$ function.

To confirm that the zero magnetic field spectrum is composed of two linearly polarized components and to precisely determine the splitting energy we have measured the energy shift versus the angle of the linear polarization in detection. The energy shift in reflectance (open squares) and PL (open triangles) shows the expected oscillatory behavior with the angle of the polarization analyser (see Figure 4(a), where the PL data has been shifted vertically by $100 \mu \mathrm{eV}$ for clarity). The data is well described using $\delta \sin ^{2}\left(\phi+\phi_{0}\right)$ (red lines in Figure 4(a)), where $\delta$ is the bright exciton FSS, $\phi$ is the angle of linear polarization detection and $\phi_{0}$ is a phase factor. Similar values of the zero field splitting are extracted from fitting the reflectance $(\delta \simeq 197 \pm 7 \mu \mathrm{eV})$ and $\mathrm{PL}(\delta \simeq 220 \pm 40 \mu \mathrm{eV})$. A detailed discussion of uncertainty estimation can be found in the supplementary information.

Theoretically the quantitative evolution of exciton fine structure exchange splitting in different perovskite phases can be derived from symmetry considerations applied to the exciton picture. ${ }^{28 / 39 / 54 / 55}$ Recently this approach has been used to provide a relation between the band structure parameters and the bright exciton fine structure splitting. $\frac{29}{2}$ The observed splittings between exciton states with dipole moment along $\mathrm{X}, \mathrm{Y}$ and $\mathrm{Z}$ can be expressed

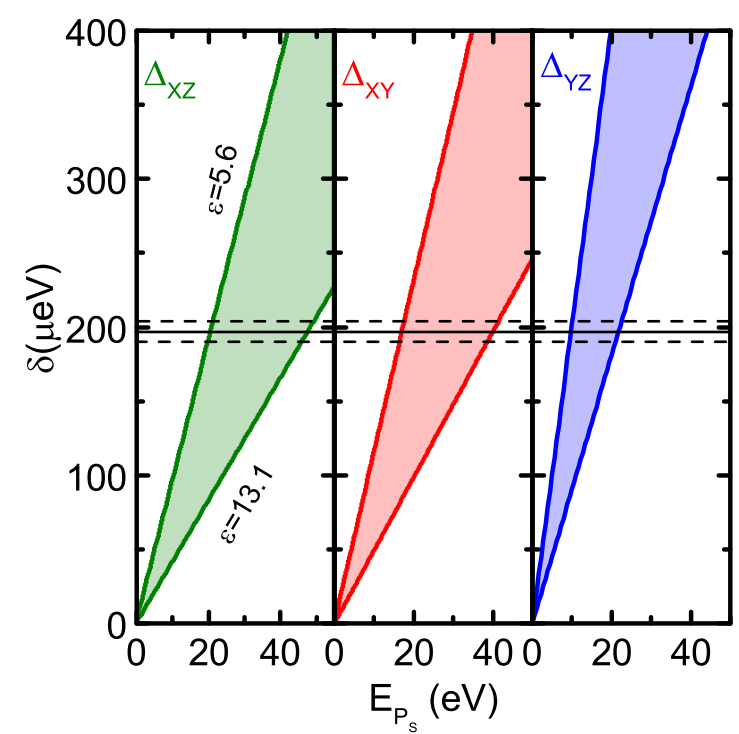

Figure 5: Calculated bright exciton fine structure splittings as a function of the Kane energy $E_{P_{S}}$ for values of the dielectric constant $\epsilon_{r}$ from 5.6 to 13.1 (see text for details). The red, blue and green colors corresponds to $\Delta_{X Y}, \Delta_{X Z}$ and $\Delta_{Y Z}$ respectively. The experimentally observed splitting is indicated by the horizontal black line. The dashed lines indicate the estimated error for the reflectance measurements. Details of the calculations and numeric values of all used parameters can be found in supplementary information.

as

$$
\begin{aligned}
& \Delta_{X Y}=2 J_{b}\left|\alpha^{2}-\beta^{2}\right| \\
& \Delta_{X Z}=2 J_{b}\left|\beta^{2}-\gamma^{2}\right| \\
& \Delta_{Y Z}=2 J_{b} \mid \alpha^{2}-\gamma^{2}
\end{aligned}
$$

where the $J_{b}$ is an exchange integral and $\alpha, \beta$ and $\gamma$ are the weights of the Bloch wave functions of electrons in the orthorombic crystal structure. The exchange integral $J_{b}$ depends on the exciton Bohr radius and Kane energy $\left(E_{P_{S}}\right)$, while $\alpha, \beta$ and $\gamma$ depend on the strength of the spin-orbit coupling and the crystal field (see detailed discussion in supplementary information).

From our magneto-optical measurements, we can extract the exciton Bohr radius $(3.8 \mathrm{~nm})$, the effective mass $(\mu=0.185)$ and the dielectric constant $\left(\epsilon_{r}=13.1\right)$ which lies between the 
accepted high (5.6-6.7) 56 and low frequency $(25-38)^{58 / 59}$ values. Such a discrepancy is typical for materials with smaller exciton radii. $\underline{60}$ Unfortunately, the Kane energy $E_{P_{S}}$ and $\alpha$ and $\beta$ parameters for $\mathrm{MAPbBr}_{3}$ are not well established. Taking into account that the spin orbit-coupling and crystal field are expected to be similar for fully inorganic and hybrid perovskites ${ }^{29[39}$ and that $\mathrm{MAPbBr}_{3}$ and $\mathrm{CsPbBr}_{3}$ have comparable band parameters ${ }^{61 / 62}$ we assume $\alpha=0.67$ and $\beta=0.57$ as in $\mathrm{CsPbBr}_{3}$. ${ }^{29}$ The estimation of the Kane energy based on 4 and 40 band $\mathbf{k} \cdot \mathbf{p}$ model gives $E_{P_{S}}=18.4 \mathrm{eV}$ and $28.4 \mathrm{eV}$ (see supplementary information for details and ref. .30$)$. In the literature, one can also find values for the Kane energy in the range of $37.1-39.9 \mathrm{eV}$ in the inorganic compounds $\mathrm{CsPbX}_{3}(\mathrm{X}=\mathrm{I}, \mathrm{Br}, \mathrm{Cl}), \stackrel{25 / 40}{\text { although these }}$ would correspond to substantially lower effective mass values. The calculated value of the splitting between different excitonic states as a function of the Kane energy are presented in Figure 5 for values of the dielectric constant in the range $\epsilon_{r}=5.6-13.1$. The predicted splitting of the bright excitonic states is in the range of a few hundreds of $\mu \mathrm{eV}$ in agreement with the experimental observation. Since all axes are equivalent in the room temperature cubic phase the orientation of the crystal axis cannot be determined after the transition to the low temperature orthorhombic phase. The accepted range of Kane energies $(\simeq 15-40 \mathrm{eV})$ is however sufficient to correspond well with the calculated splitting for any of the three possible alignments.

We have also investigated the behaviour of the FSS in magnetic field. Figure 6(a) shows PL spectra measured at $B=7 \mathrm{~T}$ in circular and linear polarization basis. In the circular basis splitting between states corresponding to $\sigma^{1}$ and $\sigma^{2}$ components of the emission is clearly visible, while both components are observed simultaneously in the linear basis. The detailed evolution of the PL peaks in the circular and linear polarization basis is shown in Figure 6(b). The fine structure splitting of the 1 s bright exciton in the orthorhombic phase, in the presence of magnetic field along the direction perpendicular to the dipole moment orientation, can be

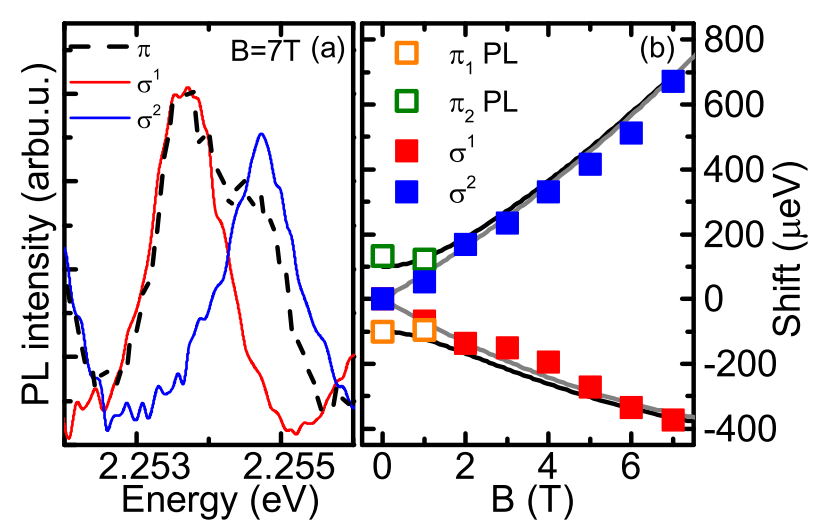

Figure 6: (a) PL spectra measured at 7T in circular polarization basis (red and blue curves) and linear basis (dashed black curve).

Dependence of PL peak position as a function of magnetic field measured in linear (open squares) and circular (full squares) polarization basis. The black lines are calculated using the exchange Hamiltonian with parameters extracted from the reflectance measurements $\delta=197 \mu \mathrm{eV}, g=2.6$ and $\sigma=3.4 \mu \mathrm{eVT}^{-1}$. The grey curve corresponding to the linear basis is calculated with $\delta=0$, i.e., neglecting the zero field splitting.

described in the $\left(\left|1^{+}\right\rangle,\left|1^{-}\right\rangle\right)$basis by the following Hamiltonian (see supplementary information and references $\left.{ }^{28 \mid 39}\right)$ :

$$
H=\frac{1}{2}\left(\begin{array}{cc}
g \mu_{B} B+2 \sigma B^{2} & \delta \\
\delta & -g \mu_{B} B+2 \sigma B^{2}
\end{array}\right)
$$

The corresponding eigenvalues and eigenstates are:

$$
E_{Y / X}= \pm \frac{1}{2} \sqrt{\left(g \mu_{B} B\right)^{2}+\delta^{2}}+\sigma B^{2}
$$

$$
|X / Y\rangle=A\left[|+1\rangle+\left(-\frac{g \mu_{B} B}{\delta} \mp \sqrt{\frac{\left(g \mu_{B} B\right)^{2}}{\delta^{2}}+1}\right)|-1\rangle\right]
$$

where $A$ is a field dependent normalizing constant. At zero magnetic field the energy difference between the states is equal to $\delta$ and the states couple to linearly polarized light (superposition of states $| \pm 1\rangle$ ). Increasing magnetic field induces a continuous change from lin- 
ear polarization to circular polarization of the excitonic transitions in agreement with experiment (see (Figure 2(c)) and Figure 6(a)). Finally, we compare the dependence of the PL peak position as a function of the magnetic field (see Fig. 6(b)) with the predictions of the exchange Hamiltonian (black lines) using the parameters extracted from reflectance studies, i.e., $\delta=200 \mu \mathrm{eV}, g=2.6$ and $\sigma=3.4 \mu \mathrm{eVT}^{-1}$. The exchange Hamiltonian provides a full quantitative description of the data. At low magnetic field, the bright FSS is determined by the zero field splitting $\delta$, and with increasing magnetic field the transition energy is increasingly dominated by the Zeeman splitting.

It is worth noting that now one can find two interpretations of the splitting of the exciton states in perovskite nanocrystals. One is solely based on crystal symmetry, exchange interaction and crystal field 28 other invokes a Rashba effect to explian FSS in fully inorganic perovskite nanocrystals. $25+27$ The Rashba effect ${ }^{25}$ has often been invoked to explain several of the unique properties of the lead halide perovskites. ${ }^{49 \mid 63}$ Despite the obvious beauty of this idea, it nevertheless remains highly controversial. For instance, the Rashba effect requires a lack of inversion symmetry or a breaking of bulk inversion symmetry, not present in centrosymmetric perovskite crystals. It was proposed that temporal symmetry breaking is provided by the cation motion, which leads to local polar fluctuations in mixed and fully inorganic perovskites. $\frac{49|65| 67}{40 w e v e r, ~}$ such vibrations of the cations should be absent at very low temperatures, ${ }^{67 / 68}$ as used for all exciton fine structure studies to date. The absence of the Rashba effect in the orthorombic phase is also consistent with circular photogalvanic measurements performed on the analogue $\mathrm{MAPbI}_{3}$ compound. 69

Another source of symmetry breaking might be attributed to surface effects, $\frac{48}{}$ which can be especially important for nanocrystals providing a possible explanation for the widely varying (200-1000 $\mathrm{eV}$ ) bright exciton FSS observed in the $\mathrm{CsPbBr}_{3}$ nanocrystals. However, in our case, both the optical methods used here, namely reflectivity and PL, probe the bulk properties of the crystal. The absorption coefficient in $\mathrm{MAPbBr}_{3}$ is of the order of $\alpha=10^{5} \mathrm{~cm}^{-1}$ at a PL excitation wavelength of $405 \mathrm{~nm}$ which corresponds to an optical absorption depth $\left(\alpha_{z}=1\right)$ of $100 \mathrm{~nm}$. The exciton diffusion will further increase the volume sampled by the PL. In the presence of a very large surface electric field the penetration depth sampled by reflectivity can fall to of the order of $20 \mathrm{~nm}(\lambda /(4 \pi n)$ where $n$ is the real part of the refractive index) ${ }^{70 / 71}$ This is still large on the scale of the lattice and exciton wavefunction $(\sim 4 \mathrm{~nm})$ and 2 times bigger than maximum surface height fluctuation amplitude (at most $10 \mathrm{~nm}$ over distances of order $100 \mu \mathrm{m}$ and much less on a wavelength scale, see SI). We have no evidence to suggest that such surface fields exist and it should be noted that the PL and reflectivity studies give identical results (within experimental error) in sign and magnitude when sampling different regions of the crystal. Therefore, both techniques measure the same property, namely the fine structure splitting of the bulk free exciton, which has not been significantly influenced by the surface.

Finally, according to models for the Rashba effect, the order of the bright and dark states should be reversed in fully inorganic perovskite nanocrystals ${ }^{[25[27]}$ which seems to be in contrast to some of the experimental reports on the PL dynamics in inorganic perovskite nanocrystals ${ }^{29 \mid 37 / 72 / 73}$ and recent direct observation of dark ground states in $\mathrm{FAPbBr}_{3}$ nanocrystals. Given all above arguments and the good quantitative agreement of the theoretical predictions based only on crystal symmetry considerations this suggests that the Rashba effect plays little role in macroscopic $\mathrm{MAPbBr}_{3}$ single crystals (and $\mathrm{FAPbBr}_{3}{ }^{31}$ ), at least for the lowest temperature phases.

It should be noted however that the excitonic band parameters, in particular the reduced effective mass, $\mu$, are significantly different from those deduced at higher energy from the magnetic field dependence of the nearly free electron Landau levels for thin films. $\frac{61}{\text { This dis- }}$ crepancy may be a hallmark of polaronic effects in perovskite crystals. ${ }^{74}$ The effective mass extracted here comes from the low field limit 
while previous reports concentrate on free electron Landau levels observed in high field limit where the motion of carriers is decoupled from lattice vibration. ${ }^{60}$ Therefore the previously extracted effective mass is lower than in the low field limits. However, this aspect is beyond the scope of this work and will be the subject of further studies.

In summary, we have observed a significant bright exciton fine structure splitting $\delta=$ $197 \mu \mathrm{eV}$ in bulk $\mathrm{MAPbBr}_{3}$. This represents an important step in the understanding of the fine structure splitting in lead-halide perovskites and perovskite based nanostructures. Using a large single crystal, we are able to exclude the influence of surface, size and anisotropic confinement on the observed bright exciton FSS giving direct access to the intrinsic properties of the bulk $\mathrm{MAPbBr}_{3}$. The observed splitting can be understood in the exciton picture combined with symmetry considerations. We show that, within this model, the observed splitting can be reasonably estimated based on band structure parameters derived from magneto-optical studies. Our work constitutes a firm base for further exciton fine structure studies in lead-halide perovskites. The presented resulst should provide valuable insight for future investigations of the different mechanisms that contribute to the exciton fine structure, such as confinement anisotropy or Rashba effect, in perovskite based nanostructures.

Acknowledgement This work was partially supported by BLAPHENE and STRABOT projects, which received funding from the IDEX Toulouse, Emergence program, "Programme des Investissements d'Avenir" under the program ANR-11-IDEX-0002-02, reference ANR-10-LABX-0037-NEXT, and by the PANCNRS collaboration within the PICS 20162018 agreement. We thank EPSRC for funding through grant EP/M05173/1. This work was supported by EPSRC also via its membership to the EMFL (Grant No. EP/N01085X/1). M.B. appreciates support from the Polish Ministry of Science and Higher Education within the Mobilnosc Plus program (grant no. 1648/MOB/V/2017/0). This work was par- tially supported by the French National Research Agency (ANR IPER-Nano2, ANR-18CE30-0023-01)

\section{Supporting Information}

PL temperature dependence, AFM surface roughens measurements, uncertainty analysis, derivation of electron-hole exchange interaction.

\section{References}

(1) Andreani, L. C.; Bassani, F. Exchange interaction and polariton effects in quantum-well excitons. Phys. Rev. B 1990, 41, 7536 .

(2) Blackwood, E.; Snelling, M.; Harley, R.; Andrews, S.; Foxon, C. Exchange interaction of excitons in GaAs heterostructures. Phys. Rev. B 1994, 50, 14246.

(3) Salmassi, B. R.; Bauer, G. E. Exchange interaction in type-II quantum wells. Phys. Rev. B 1989, 39, 1970.

(4) Gammon, D.; Snow, E.; Shanabrook, B.; Katzer, D.; Park, D. Fine structure splitting in the optical spectra of single GaAs quantum dots. Phys. Rev. Lett. 1996, 76, 3005 .

(5) Bayer, M.; Kuther, A.; Forchel, A.; Gorbunov, A.; Timofeev, V.; Schäfer, F.; Reithmaier, J.; Reinecke, T.; Walck, S. Electron and hole $\mathrm{g}$ factors and exchange interaction from studies of the exciton fine structure in In 0.60 Ga 0.40 As quantum dots. Phys. Rev. Lett. 1999, 82, 1748.

(6) Bayer, M.; Ortner, G.; Stern, O.; Kuther, A.; Gorbunov, A.; Forchel, A.; Hawrylak, P.; Fafard, S.; Hinzer, K.; Reinecke, T. et al. Fine structure of neutral and charged excitons in self-assembled In (Ga) As/(Al) GaAs quantum dots. Phys. Rev. B 2002, 65, 195315. 
(7) Puls, J.; Rabe, M.; Wünsche, H.-J.; Henneberger, F. Magneto-optical study of the exciton fine structure in self-assembled CdSe quantum dots. Phys. Rev. B 1999, 60, R16303.

(8) Besombes, L.; Kheng, K.; Martrou, D. Exciton and biexciton fine structure in single elongated islands grown on a vicinal surface. Phys. Rev. Lett. 2000, 85, 425.

(9) Nirmal, M.; Norris, D. J.; Kuno, M.; Bawendi, M. G.; Efros, A. L.; Rosen, M. Observation of the" dark exciton" in CdSe quantum dots. Phys. Rev. Lett. 1995, 75, 3728.

(10) Chen, Y.; Gil, B.; Lefebvre, P.; Mathieu, H. Exchange effects on excitons in quantum wells. Phys. Rev. B 1988, 37, 6429 .

(11) Bester, G.; Nair, S.; Zunger, A. Pseudopotential calculation of the excitonic fine structure of million-atom self-assembled In 1- x Ga x A s/G a A s quantum dots. Phys. Rev. B 2003, 67, 161306.

(12) Mrowiński, P.; Musial, A.; Maryński, A.; Syperek, M.; Misiewicz, J.; Somers, A.; Reithmaier, J.; Hofling, S.; Sek, G. Magnetic field control of the neutral and charged exciton fine structure in single quantum dashes emitting at $1.55 \mu \mathrm{m}$. Appl. Phys. Lett. 2015, 106, 053114.

(13) Mrowiński, P.; Zieliński, M.; Świderski, M.; Misiewicz, J.; Somers, A.; Reithmaier, J.; Höfling, S.; Sk, G. Excitonic fine structure and binding energies of excitonic complexes in single InAs quantum dashes. Phys. Rev. B 2016, 94, 115434.

(14) Li, X.; Wu, Y.; Steel, D.; Gammon, D.; Stievater, T.; Katzer, D.; Park, D.; Piermarocchi, C.; Sham, L. An all-optical quantum gate in a semiconductor quantum dot. Science 2003, 301, 809-811.

(15) Bonadeo, N. H.; Erland, J.; Gammon, D.; Park, D.; Katzer, D.; Steel, D. Coherent optical control of the quantum state of a single quantum dot. Science 1998, 282, 1473-1476.

(16) Stevenson, R. M.; Young, R. J.; Atkinson, P.; Cooper, K.; Ritchie, D. A.; Shields, A. J. A semiconductor source of triggered entangled photon pairs. Nature 2006, 439, 179-182.

(17) Bennett, A.; Pooley, M.; Stevenson, R.; Ward, M.; Patel, R.; de La Giroday, A. B.; Sköld, N.; Farrer, I.; Nicoll, C.; Ritchie, D. et al. Electric-field-induced coherent coupling of the exciton states in a single quantum dot. Nat. Phys. 2010, 6, 947-950.

(18) Benson, O.; Santori, C.; Pelton, M.; Yamamoto, Y. Regulated and entangled photons from a single quantum dot. Phys. Rev. Lett. 2000, 84, 2513.

(19) Müller, M.; Bounouar, S.; Jöns, K. D.; Glässl, M.; Michler, P. On-demand generation of indistinguishable polarizationentangled photon pairs. Nat. Photonics 2014, 8, 224-228.

(20) Dousse, A.; Suffczyński, J.; Beveratos, A.; Krebs, O.; Lemaître, A.; Sagnes, I.; Bloch, J.; Voisin, P.; Senellart, P. Ultrabright source of entangled photon pairs. Nature 2010, 466, 217-220.

(21) Bouwmeester, D.; Pan, J.-W.; Mattle, K.; Eibl, M.; Weinfurter, H.; Zeilinger, A. Experimental quantum teleportation. Nature 1997, 390, 575-579.

(22) Briegel, H.-J.; Dür, W.; Cirac, J. I.; Zoller, P. Quantum repeaters: the role of imperfect local operations in quantum communication. Phys. Rev. Lett. 1998, 81,5932

(23) Gisin, N.; Ribordy, G.; Tittel, W.; Zbinden, H. Quantum cryptography. Rev. Mod. Phys. 2002, 74, 145.

(24) Fu, H.; Wang, L.-W.; Zunger, A. Excitonic exchange splitting in bulk semiconductors. Phys. Rev. B 1999, 59, 5568. 
(25) Becker, M. A.; Vaxenburg, R.; Nedelcu, G.; Sercel, P. C.; Shabaev, A.; Mehl, M. J.; Michopoulos, J. G.; Lambrakos, S. G.; Bernstein, N.; Lyons, J. L. et al. Bright triplet excitons in caesium lead halide perovskites. Nature 2018, 553, 189.

(26) Isarov, M.; Tan, L. Z.; Bodnarchuk, M. I.; Kovalenko, M. V.; Rappe, A. M.; Lifshitz, E. Rashba effect in a single colloidal $\mathrm{CsPbBr} 3$ perovskite nanocrystal detected by magneto-optical measurements. Nano Lett. 2017, 17, 5020-5026.

(27) Sercel, P. C.; Lyons, J. L.; Wickramaratne, D.; Vaxenburg, R.; Bernstein, N.; Efros, A. L. Exciton Fine Structure in Perovskite Nanocrystals. Nano Lett. 2019, 19, 4068-4077.

(28) Fu, M.; Tamarat, P.; Huang, H.; Even, J.; Rogach, A. L.; Lounis, B. Neutral and charged exciton fine structure in single lead halide perovskite nanocrystals revealed by magneto-optical spectroscopy. Nano Lett. 2017, 17, 2895-2901.

(29) Ramade, J.; Andriambariarijaona, L. M.; Steinmetz, V.; Goubet, N.; Legrand, L.; Barisien, T.; Bernardot, F.; Testelin, C.; Lhuillier, E.; Bramati, A. et al. Fine structure of excitons and electron-hole exchange energy in polymorphic $\mathrm{CsPbBr} 3$ single nanocrystals. Nanoscale 2018, 10, 6393-6401.

(30) Aich, R. B.; Saïdi, I.; Radhia, S. B.; Boujdaria, K.; Barisien, T.; Legrand, L.; Bernardot, F.; Chamarro, M.; Testelin, C. Bright-Exciton Splittings in Inorganic Cesium Lead Halide Perovskite Nanocrystals. Phys. Rev. Appl. 2019, 11, 034042.

(31) Tamarat, P.; Bodnarchuk, M. I.; Trebbia, J.-B.; Erni, R.; Kovalenko, M. V.; Even, J.; Lounis, B. The ground exciton state of formamidinium lead bromide perovskite nanocrystals is a singlet dark state. Nat. Mater. 2019, 18, 717-724.
(32) Even, J.; Pedesseau, L.; Jancu, J.-M.; Katan, C. Importance of spin-orbit coupling in hybrid organic/inorganic perovskites for photovoltaic applications. $J$. Phys. Chem. Lett. 2013, 4, 2999-3005.

(33) Even, J.; Pedesseau, L.; Jancu, J.-M.; Katan, C. DFT and $\mathrm{k} \cdot \mathrm{p}$ modelling of the phase transitions of lead and tin halide perovskites for photovoltaic cells. Phys. Status Solidi RRL 2014, 8, 31-35.

(34) Brivio, F.; Butler, K. T.; Walsh, A.; Van Schilfgaarde, M. Relativistic quasiparticle self-consistent electronic structure of hybrid halide perovskite photovoltaic absorbers. Phys. Rev. B 2014, 89, 155204.

(35) Endres, J.; Egger, D. A.; Kulbak, M.; Kerner, R. A.; Zhao, L.; Silver, S. H.; Hodes, G.; Rand, B. P.; Cahen, D.; Kronik, L. et al. Valence and Conduction Band Densities of States of Metal Halide Perovskites: A Combined ExperimentalTheoretical Study. J. Phys. Chem. Lett. 2016, 7, 2722-2729.

(36) Poglitsch, A.; Weber, D. Dynamic disorder in methylammoniumtrihalogenoplumbates (II) observed by millimeterwave spectroscopy. J. Chem. Phys. 1987, 87, 6373-6378.

(37) Chen, C.; Hu, X.; Lu, W.; Chang, S.; Shi, L.; Li, L.; Zhong, H.; Han, J.B. Elucidating the phase transitions and temperature-dependent photoluminescence of MAPbBr3 single crystal. $J$. Phys. D: Appl. Phys. 2018, 51, 045105.

(38) Odenthal, P.; Talmadge, W.; Gundlach, N.; Wang, R.; Zhang, C.; Sun, D.; Yu, Z.-G.; Vardeny, Z. V.; Li, Y. S. Spinpolarized exciton quantum beating in hybrid organic-inorganic perovskites. Nat. Phys. 2017,

(39) Yu, Z. Effective-mass model and magnetooptical properties in hybrid perovskites. Sci. Rep. 2016, 6, 28576. 
(40) Nestoklon, M.; Goupalov, S.; Dzhioev, R.; Ken, O.; Korenev, V.; Kusrayev, Y. G.; Sapega, V.; de Weerd, C.; Gomez, L.; Gregorkiewicz, T. et al. Optical orientation and alignment of excitons in ensembles of inorganic perovskite nanocrystals. Phys. Rev. B 2018, 97, 235304.

(41) Yin, C.; Chen, L.; Song, N.; Lv, Y.; $\mathrm{Hu}$, F.; Sun, C.; William, W. Y.; Zhang, C.; Wang, X.; Zhang, Y. et al. Bright-Exciton Fine-Structure Splittings in Single Perovskite Nanocrystals. Phys. Rev. Lett. 2017, 119, 026401.

(42) Pfingsten, O.; Klein, J.; Protesescu, L.; Bodnarchuk, M. I.; Kovalenko, M. V.; Bacher, G. Phonon Interaction and Phase Transition in Single Formamidinium Lead Bromide Quantum Dots. Nano Lett. 2018, 18, 4440-4446.

(43) Raino, G.; Nedelcu, G.; Protesescu, L.; Bodnarchuk, M. I.; Kovalenko, M. V.; Mahrt, R. F.; Stoferle, T. Single cesium lead halide perovskite nanocrystals at low temperature: fast single-photon emission, reduced blinking, and exciton fine structure. ACS nano 2016, 10, 2485-2490.

(44) Ramade, J.; Andriambariarijaona, L. M.; Steinmetz, V.; Goubet, N.; Legrand, L.; Barisien, T.; Bernardot, F.; Testelin, C.; Lhuillier, E.; Bramati, A. et al. Excitonphonon coupling in a $\mathrm{CsPbBr} 3$ single nanocrystal. Appl. Phys. Lett. 2018, 112, 072104 .

(45) Zieliński, M. Excitonic fine structure of elongated InAs/InP quantum dots. Phys. Rev. B 2013, 88, 155319.

(46) Seguin, R.; Schliwa, A.; Rodt, S.; Pötschke, K.; Pohl, U.; Bimberg, D. Size-dependent fine-structure splitting in self-organized InAs/GaAs quantum dots. Phys. Rev. Lett. 2005, 95, 257402.

(47) Ben Aich, R.; Saidi, I.; Ben Radhia, S.; Boujdaria, K.; Barisien, T.; Legrand, T.; Bernandot, F.; Chamarro, M.; Testelin, C.
Article in preparation. Submitted to Phys. Rev. Appl.

(48) Niesner, D.; Wilhelm, M.; Levchuk, I.; Osvet, A.; Shrestha, S.; Batentschuk, M.; Brabec, C.; Fauster, T. Giant Rashba Splitting in $\mathrm{CH} 3 \mathrm{NH} 3 \mathrm{PbBr} 3$ OrganicInorganic Perovskite. Phys. Rev. Lett. 2016, 11\%, 126401.

(49) Mosconi, E.; Etienne, T.; De Angelis, F. Rashba Band Splitting in Organohalide Lead Perovskites: Bulk and Surface Effects. J. Phys. Chem. Lett. 2017,

(50) Swarnkar, A.; Marshall, A. R.; Sanehira, E. M.; Chernomordik, B. D.; Moore, D. T.; Christians, J. A.; Chakrabarti, T.; Luther, J. M. Quantum dot-induced phase stabilization of $\alpha$-CsPbI3 perovskite for high-efficiency photovoltaics. Science 2016, 354, 92-95.

(51) Nayak, P. K.; Moore, D. T.; Wenger, B.; Nayak, S.; Haghighirad, A. A.; Fineberg, A.; Noel, N. K.; Reid, O. G.; Rumbles, G.; Kukura, P. et al. Mechanism for rapid growth of organic-inorganic halide perovskite crystals. Nat. Commun. 2016, r, 13303.

(52) Tilchin, J.; Dirin, D. N.; Maikov, G. I.; Sashchiuk, A.; Kovalenko, M. V.; Lifshitz, E. Hydrogen-like Wannier-Mott Excitons in Single Crystal of Methylammonium Lead Bromide Perovskite. ACS nano 2016, 10, 6363-6371.

(53) Wright, A. D.; Verdi, C.; Milot, R. L.; Eperon, G. E.; Pérez-Osorio, M. A.; Snaith, H. J.; Giustino, F.; Johnston, M. B.; Herz, L. M. Electron-phonon coupling in hybrid lead halide perovskites. Nat. Commun. 2016, \%, 11755.

(54) Kataoka, T.; Kondo, T.; Ito, R.; Sasaki, S.; Uchida, K.; Miura, N. Magneto-optical study on excitonic spectra in (C $6 \mathrm{H} 13 \mathrm{NH} 3) 2 \mathrm{PbI}$ 4. Phys. Rev. $B$ 1993, 47, 2010. 
(55) Tanaka, K.; Takahashi, T.; Kondo, T.; Umeda, K.; Ema, K.; Umebayashi, T.; Asai, K.; Uchida, K.; Miura, N. Electronic and excitonic structures of inorganicorganic perovskite-type quantum-well crystal (C4H9NH3) 2PbBr4. Jpn. J. Appl. Phys. 2005, 44, 5923.

(56) Ndione, P. F.; Li, Z.; Zhu, K. Effects of alloying on the optical properties of organicinorganic lead halide perovskite thin films. Journal of Materials Chemistry C 2016, 4, 7775-7782.

(57) Valverde-Chávez, D. A.; Ponseca, C. S.; Stoumpos, C. C.; Yartsev, A.; Kanatzidis, M. G.; Sundström, V.; Cooke, D. G. Intrinsic femtosecond charge generation dynamics in single crystal $\mathrm{CH} \quad 3 \quad \mathrm{NH} \quad 3 \mathrm{PbI} 3$. Energy Environ. Sci. 2015, 8, 3700-3707.

(58) Zhao, D.; Skelton, J. M.; Hu, H.; La-o Vorakiat, C.; Zhu, J.-X.; Marcus, R. A.; Michel-Beyerle, M.-E.; Lam, Y. M.; Walsh, A.; Chia, E. E. Low-frequency optical phonon modes and carrier mobility in the halide perovskite $\mathrm{CH} 3 \mathrm{NH} 3 \mathrm{PbBr} 3$ using terahertz time-domain spectroscopy. Appl. Phys. Lett. 2017, 111, 201903.

(59) Anusca, I.; Balčiūnas, S.; Gemeiner, P.; Svirskas, Š.; Sanlialp, M.; Lackner, G.; Fettkenhauer, C.; Belovickis, J.; Samulionis, V.; Ivanov, M. et al. Dielectric response: Answer to many questions in the methylammonium lead halide solar cell absorbers. Adv. Energy Mater. 2017, 7, 1700600 .

(60) Miura, N. Physics of semiconductors in high magnetic fields; Oxford University Press: New York, 2008; Vol. 15.

(61) Galkowski, K.; Mitioglu, A.; Miyata, A.; Plochocka, P.; Portugall, O.; Eperon, G. E.; Wang, J. T.-W.; Stergiopoulos, T.; Stranks, S. D.; Snaith, H. J. et al. Determination of the exciton binding energy and effective masses for methylammonium and formamidinium lead tri- halide perovskite semiconductors. Energy Environ. Sci. 2016, 9, 962-970.

(62) Yang, Z.; Surrente, A.; Galkowski, K.; Miyata, A.; Portugall, O.; Sutton, R. J.; Haghighirad, A.; Snaith, H. J.; Maude, D. K.; Plochocka, P. et al. Impact of the halide cage on the electronic properties of fully inorganic cesium lead halide perovskites. ACS Energy Lett. 2017, 2, 1621-1627.

(63) Kepenekian, M.; Even, J. Rashba and Dresselhaus Couplings in Halide Perovskites: Accomplishments and Opportunities for Spintronics and SpinOrbitronics. J. Phys. Chem. Lett. 2017, 8, 3362-3370.

(64) Zheng, F.; Tan, L. Z.; Liu, S.; Rappe, A. M. Rashba spin-orbit coupling enhanced carrier lifetime in CH3NH3PbI3. Nano Lett. 2015, 15, 7794-7800.

(65) Etienne, T.; Mosconi, E.; De Angelis, F. Dynamical Origin of the Rashba Effect in Organohalide Lead Perovskites: A Key to Suppressed Carrier Recombination in Perovskite Solar Cells? J. Phys. Chem. Lett. 2016, 7, 1638-1645.

(66) Quarti, C.; Mosconi, E.; De Angelis, F. Interplay of orientational order and electronic structure in methylammonium lead iodide: implications for solar cell operation. Chem. Mater. 2014, 26, 6557-6569.

(67) Yaffe, O.; Guo, Y.; Tan, L. Z.; Egger, D. A.; Hull, T.; Stoumpos, C. C.; Zheng, F.; Heinz, T. F.; Kronik, L.; Kanatzidis, M. G. et al. Local polar fluctuations in lead halide perovskite crystals. Phys. Rev. Lett. 2017, 118, 136001.

(68) Dar, M. I.; Jacopin, G.; Meloni, S.; Mattoni, A.; Arora, N.; Boziki, A.; Zakeeruddin, S. M.; Rothlisberger, U.; Grätzel, M. Origin of unusual bandgap shift and dual emission in organic-inorganic lead halide perovskites. Sci. Adv. 2016, 2, e1601156. 
(69) Niesner, D.; Hauck, M.; Shrestha, S.; Levchuk, I.; Matt, G. J.; Osvet, A.; Batentschuk, M.; Brabec, C.; Weber, H. B.; Fauster, T. Structural fluctuations cause spin-split states in tetragonal (CH3NH3) $\mathrm{PbI} 3$ as evidenced by the circular photogalvanic effect. Proceedings of the National Academy of Sciences 2018, 115, 95099514 .

(70) Yang, Y.; Yan, Y.; Yang, M.; Choi, S.; Zhu, K.; Luther, J. M.; Beard, M. C. Low surface recombination velocity in solutiongrown $\mathrm{CH} 3 \mathrm{NH} 3 \mathrm{PbBr} 3$ perovskite single crystal. Nat. Commun. 2015, 6, 7961.

(71) Sabbah, A.; Riffe, D. M. Femtosecond pump-probe reflectivity study of silicon carrier dynamics. Phys. Rev. B 2002, 66, 165217.

(72) Canneson, D.; Shornikova, E. V.; Yakovlev, D. R.; Rogge, T.; Mitioglu, A. A.; Ballottin, M. V.; Christianen, P. C.; Lhuillier, E.; Bayer, M.; Biadala, L. Negatively charged and dark excitons in $\mathrm{CsPbBr} 3$ perovskite nanocrystals revealed by high magnetic fields. Nano Lett. 2017, 17, 6177-6183.

(73) Xu, K.; Vliem, J. F.; Meijerink, A. LongLived Dark Exciton Emission in MnDoped CsPbCl3 Perovskite Nanocrystals. J. Mater. Chem. C 2018, 123, 979-984.

(74) Zhu, X.-Y.; Podzorov, V. Charge carriers in hybrid organic-inorganic lead halide perovskites might be protected as large polarons. J. Phys. Chem. Lett. 2015, 6, 4758-4761.

(75) Schlipf, M.; Poncé, S.; Giustino, F. Carrier lifetimes and polaronic mass enhancement in the hybrid halide perovskite $\mathrm{CH} 3 \mathrm{NH}$ 3 PbI 3 from multiphonon Fröhlich coupling. Phys. Rev. Lett. 2018, 121, 086402.

(76) Sendner, M.; Nayak, P. K.; Egger, D. A.; Beck, S.; Müller, C.; Epding, B.; Kowalsky, W.; Kronik, L.; Snaith, H. J.;
Pucci, A. et al. Optical phonons in methylammonium lead halide perovskites and implications for charge transport. Mater. Horiz. 2016, 3, 613-620.

(77) Cinquanta, E.; Meggiolaro, D.; Motti, S. G.; Gandini, M.; Alcocer, M. J.; Akkerman, Q. A.; Vozzi, C.; Manna, L.; De Angelis, F.; Petrozza, A. et al. Ultrafast THz Probe of Photoinduced Polarons in Lead-Halide Perovskites. Phys. Rev. Lett. 2019, 122, 166601. 
Graphical TOC Entry

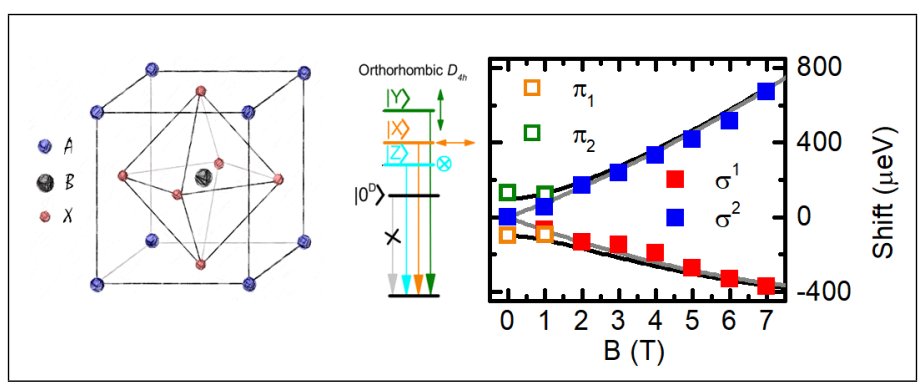

